PAPER

\title{
Is inflammation important in early PPMS? a longitudinal MRI study
}

\author{
G T Ingle, J Sastre-Garriga, D H Miller, A J Thompson
}

J Neurol Neurosurg Psychiatry 2005;76:1255-1258. doi: 10.1136/jnnp.2004.036590

See end of article for authors' affiliations .....................

Correspondence to: Professor Alan J Thompson, Institute of Neurology, University College London, Queen Square, London WCIN 3BG, UK; a.thompson@ion.ucl.ac.uk

Received 14 January 2004 In revised form

27 October 2004

Accepted

10 December 2004
Background: Magnetic resonance imaging (MRI) studies in primary progressive multiple sclerosis (PPMS) have shown a reduced frequency of enhancement with the contrast agent gadolinium-DTPA (Gd-DTPA), in comparison with relapsing-remitting multiple sclerosis (RRMS), and it has been suggested that there may be a less important role for inflammation in its pathogenesis. However, the earliest clinical stages of PPMS have not been studied and thus it has not been possible to exclude the existence of an early inflammatory phase.

Objective: To study the presence, characteristics, and implications of inflammation in early PPMS.

Methods: 45 patients with a mean disease duration of 3.3 years had triple dose Gd enhanced MRI, expanded disability status scale (EDSS), and multiple sclerosis functional composite (MSFC) assessments at baseline. Repeat MRI was done at 1 and 2 months in 24 patients, and at 6 months in 38.

Results: Enhancing brain lesions were present in $42 \%$ of patients at baseline but enhancing cord lesions were uncommon (7\%); $85 \%$ of enhancing lesions enhanced for one month or less. Patients with enhancing lesions had greater disability (EDSS, $p=0.027$; MSFC, $p=0.026$ ) and more MRI abnormalities (greater T2 load, $p=0.008$; greater T1 hypointensity load, $p=0.001$; and reduced partial brain volume, $p=0.012$ ) than those without enhancement. Enhancement at 6 months was seen in $32 \%$ of patients and was restricted to a subset of patients who enhanced at baseline.

Conclusions: Enhancement is present in some cases of early PPMS and is associated with greater disease impact in terms of both clinical and MRI measures.
M ultiple sclerosis follows a progressive rather than a relapsing course from onset in approximately 15\% of cases. This is termed primary progressive multiple sclerosis (PPMS ). ${ }^{1}$ Patients with PPMS differ from those with relapsing-remitting multiple sclerosis (RRMS) in being older and having a more equal sex incidence. ${ }^{2}$ On magnetic resonance imaging (MRI), they have fewer, smaller lesions and a lower rate of new lesion formation. In addition, enhancement with gadolinium-DTPA (Gd-DTPA) on Tl weighted magnetic resonance imaging (MRI), an indicator of blood-brain barrier breakdown which is associated with CNS inflammation,,$^{3-5}$ is relatively rare in PPMS..$^{6-8}$ With triple dose Gd-DTPA it may be possible to detect more enhancing lesions but the overall frequency of enhancement is still less than in other subtypes of multiple sclerosis. ${ }^{9}{ }^{10}$ In RRMS, enhancing lesions have a significant association with clinical relapse. ${ }^{11}$ In PPMS, the clinical significance of enhancement is less clear. MRI evidence suggesting a lesser role for inflammation in PPMS is supported by pathological studies. ${ }^{12}$ This may explain the negative results of treatment trials in PPMS with agents known to suppress inflammation such as interferon beta and glatiramer acetate. ${ }^{13}{ }^{14}$

In RRMS, there is evidence that the predominant pathological process operating early in the condition differs from those that occur later; in early phases, inflammation is predominant; in later phases, degeneration. ${ }^{15}$ If a similar pattern were to exist in PPMS this would have important implications for treatment strategies. Our aim in this study was to assess the presence, characteristics, and consequences of inflammation in the early stages of PPMS. We examined a cohort of patients seen within five years of symptom onset and sought to answer the following questions:

- Is there more enhancement in early PPMS than in established disease?
- How do enhancing lesions evolve?

- Do PPMS patients with enhancement differ from those without?

- Do the clinical and MRI associations of enhancement provide any insight into the pathological role of inflammation in PPMS?

\section{METHODS}

A new cohort of patients with PPMS with a disease duration of five years or less was recruited from referrals to the National Hospital and from a direct appeal to neurologists in the surrounding hospitals. Patients were asked to take part in a three year MRI and clinical study involving scans at baseline, one month, and two months, and then at six monthly intervals. Confirmation that patients were within five years of first symptom onset was determined from clinical records and by interview. In all cases patients had a clinical diagnosis of PPMS and had oligoclonal bands in their cerebrospinal fluid which were not seen in serum. According to recent diagnostic criteria for PPMS $^{16}$ at baseline, 43 patients $(95 \%)$ had definite PPMS and two had probable PPMS.

Clinical examination consisted of a neurological examination with calculation of the expanded disability status score (EDSS). The nine hole peg test, timed 25 foot walk, and paced auditory serial addition test (PASAT) were also measured and used to calculate the multiple sclerosis functional composite (MSFC). ${ }^{17}$

Abbreviations: EDSS, expanded disability status scale; MSFC, multiple sclerosis functional composite; PASAT, paced auditory serial addition test; PPMS, primary progressive multiple sclerosis; RRMS, relapsingremitting multiple sclerosis 
Imaging of brain and spinal cord was carried out at baseline and at one and two months and again at a follow up visit at six months. Cerebral lesion loads were quantified, together with partial brain and ventricular volume. The same scanner (Signa 1.5T system, General Electric, Milwaukee, Wisconsin, USA) was used throughout the study. Each patient underwent Tl imaging of the brain and cord before and immediately after administration of triple dose Gd-DTPA at a dose of $0.3 \mathrm{mmol} / \mathrm{kg}$. T2 weighted spin echo imaging of the brain (T2: time of repetition (TR) $3000 \mathrm{~ms}$, time of echo (TE) 15/90 ms; Tl: TR $600 \mathrm{~ms}$, TE $20 \mathrm{~ms}$ ) was also carried out after Gd-DTPA administration. All sequences were acquired as contiguous $5 \mathrm{~mm}$ thick axial slices. All enhancing lesions were identified by a single experienced rater (DHM) and on follow up the scans were classified as new (first appearance) or persistent (seen on previous scan). Image analysis was carried out at a SUN workstation using a local thresholding technique with manual editing. $\mathrm{T} 2$ and $\mathrm{Tl}$ hypointensity volumes were calculated with reference to marked hard copies.

The measure of partial brain volume, reflecting atrophy, was calculated using methods described previously. ${ }^{18} 19$ Brain volume was measured by extracting the brain images from the skull using a computer algorithm and by measuring the volume occupied by four $5 \mathrm{~mm}$ slices, the most caudal being at the level of the velum interpositum cerebri. Ventricular volumes were obtained by analysing the Tl weighted images using an interactive image analysis package (MIDAS). ${ }^{20}$ Whole brain regions were obtained using a semiautomated iterative morphological technique originally developed for three dimensional volumetric scans. Mean signal intensity over these brain regions was calculated. Ventricular regions were outlined using a thresholding technique with the ventricular-brain boundary being set at $60 \%$ of the whole brain signal intensity. The ventricular region consisted of the lateral ventricles including the temporal horn but excluding the third and fourth ventricles. High signal structures within the ventricles-for example, blood vessels-were excluded. Ventricular volumes were automatically calculated from the outlined regions by multiplying total area outlined by slice thickness.

\section{Statistics}

Non-parametric tests of statistical significance were used throughout. Cross sectional comparisons between groups were carried out using the Mann-Whitney $U$ test, and changes in MRI and clinical measures over time were assessed using the Wilcoxon signed rank test. Correlations were assessed using Spearman's rank correlation coefficient.

\section{RESULTS}

Forty five patients were enrolled into the study and 24 of these agreed to attend for three initial scans done at monthly intervals. Follow up data on 38 patients were available at six months (mean (SD), 6.1 (1.2) months). For seven patients no follow up data were available. This was because of death from a non-multiple sclerosis related cause (one case), dislike of MRI (one case), intercurrent non-multiple sclerosis related surgical illness (one case), personal reasons (three cases), and non-residency in United Kingdom (one case).

\section{Baseline, clinical}

The 45 patients studied at baseline comprised 26 men and 19 women with an age range of 22 to 65 years. Mean (SD) age was 45.2 (10.9) years and disease duration was $3.3(0.9)$ years. Thirty seven of the 45 cases presented with a cord syndrome (82\%). The median EDSS was 4.5 (range 1.5 to 7.0 ) and the mean (SD) MSFC was -0.8 (1.5). EDSS and MSFC were highly correlated $(r=0.7, \mathrm{p}<0.001)$. Younger patients had shorter disease duration $(r=0.5, \mathrm{p}=0.001)$. Patients with cord and non-cord presentations did not differ significantly in age, disease duration, or EDSS but there was a trend to greater disability in patients with cord presentations as measured by the MSFC $(p=0.05)$.

\section{Baseline MRI}

Of the 45 patients studied at baseline (month 0), 19 (42\%) had at least one enhancing lesion (fig lA). In patients with enhancement, the mean (SD) number of enhancing lesions seen was 2.4 (2.1) and the average enhancing lesion volume was $0.3(0.2) \mathrm{ml}$. Three patients had enhancing cord lesions $(7 \%)$ at baseline (fig 1B), all of whom had enhancing cerebral lesions. The number of enhancing lesions correlated inversely with age $(r=-0.4, \mathrm{p}=0.006)$ and directly with $\mathrm{T} 2$ load $(r=0.6, \mathrm{p}<0.001)$. When divided by the presence or absence of enhancement (table 1), patients with enhancement had greater disability (EDSS, $\mathrm{p}=0.0027$; MSFC, $\mathrm{p}=0.026$ ), higher $\mathrm{T} 2$ and $\mathrm{T} 1$ hypointensity loads $(\mathrm{p}=0.008$ and $\mathrm{p}=0.001$, respectively), and a trend to greater atrophy as assessed by partial brain volume $(p=0.012)$. Gd-DTPA enhancement was seen in $35 \%$ of patients with cord symptoms at initial presentation and in $75 \%$ of those without. This difference was not significant.

\section{Monthly MRI}

Twenty four patients (16 male, eight female) attended for monthly serial examinations at baseline. Within this group, 13 patients had enhancement on the baseline scan and eight $(62 \%)$ enhanced at every time point (fig 2 ). Of the 11 patients not showing enhancement at baseline, none showed subsequent enhancement.

The duration of enhancement of individual lesions over the first three months is shown graphically in fig 2. Of the 25 enhancing lesions that were seen at baseline, five were still enhancing at month $\mathrm{l}$ and only one was still enhancing at month 2. Thirteen new lesions were seen at month 1 and of these two were still enhancing at month 2 . Thus 11 of the 13 new enhancing lesions (85\%) enhanced between months 1 and 2 .

Serial examination at months 1 and 2 confirmed the low frequency of spinal cord enhancement seen at month 0 . From a total of 72 monthly cord observations, only four instances of cord enhancement were observed (6\%). Of two patients with enhancing spinal cord lesions at baseline, one showed further spinal cord enhancement at month 1 and month 2, while the other enhanced at baseline only. In each case cerebral enhancing lesions were also present.

\section{Six month follow up}

The 38 patients ( 23 male and 15 female) on whom six month data were available did not differ from the baseline cohort. At six months, the scans of 12 patients showed enhancement

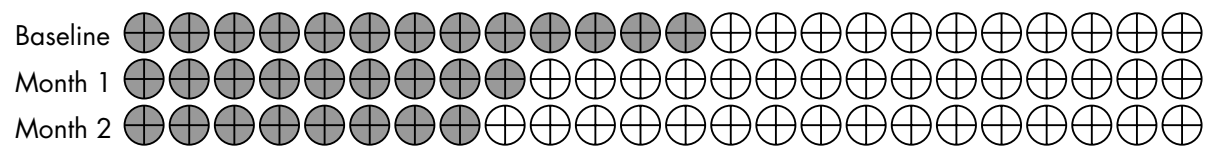

Figure 1 Graphical representation of the pattern of cerebral and spinal cord enhancement by patient at the baseline, month 1, and month 2 time points. Patients with enhancement at that time point are shown in dark grey. 
Table 1 Comparison of clinical and magnetic resonance imaging features of patients with and without enhancing lesions

\begin{tabular}{llll}
\hline Variable & Enhancing (19) & Non-enhancing (26) & p Value \\
\hline Age (years) & $42.0(11.4)$ & $47.5(10.1)$ & 0.084 \\
Sex (n) & M 11; F 8 & M 16; F 10 & \\
Disease duration (years) & $3.2(0.8)$ & $3.4(0.9)$ & 0.355 \\
Presentation (n) & Cord 13; non-cord 6 & Cord 24; non-cord 2 & \\
EDSS & $6.0(3.5$ to 7.0) & $4.3(1.5$ to 7.0) & $0.027^{*}$ \\
MSFC & $-1.3(1.8)$ & $-0.4(1.1)$ & $0.026^{*}$ \\
T2 load (ml) & $21.7(24.6)$ & $9.5(19.5)$ & $0.008^{* *}$ \\
T1 load (ml) & $7.1(9.8)$ & $3.2(9.6)$ & $0.001^{* *}$ \\
Ventricular volume (ml) & $22.4(19.7)$ & $20.6(18.0)$ & 0.696 \\
Partial brain volume (ml) & $247.5(23.5)$ & $267.0(20.3)$ & $0.012^{*}$ \\
Whole brain volume (ml) & $1106.1(135.6)$ & $1197.4(137.8)$ & $0.050^{*}$ \\
\hline Values are mean (SD) or mean (range) unless stated otherwise. & & \\
** $\mathrm{p} \leqslant 0.005 ;{ }^{*} \mathrm{p} \leqslant 0.05$. & & \\
EDSS, expanded disability status scale; F, female; M, male; MSFC, multiple sclerosis functional composite.
\end{tabular}

$(32 \%)$ and all of these had enhanced at baseline. No patient without initial enhancement showed enhancement at six months. Thus enhancement status did not change in 34 of 38 cases $(89 \%)$, and the presence of enhancement at baseline was strongly associated with enhancement at six months $(r=0.8, \mathrm{p}<0.001)$. The one patient with an enhancing cord lesion at six months (3\%) also had an enhancing brain lesion at six months and an enhancing cord and brain lesion at baseline. None of the enhancing lesions seen on the initial monthly scans was still enhancing at six months; $64 \%$ became T2 lesions and 39\% became T1 lesions. Fifty five per cent of the lesions that became T2 lesions (and 35\% of all enhancing lesions) also became Tl lesions.

Over six months, the only MRI measure that showed a significant increase was mean T2 load $(14.4 \mathrm{ml}$ to $15.9 \mathrm{ml}$, $\mathrm{p}=0.028$ ). The presence of enhancement at baseline was not associated with greater or lesser change in any MRI or clinical measure over six months.

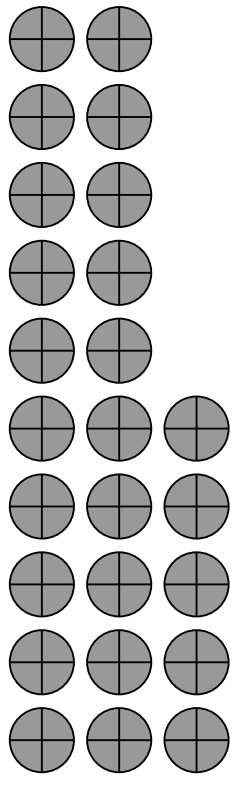

Baseline

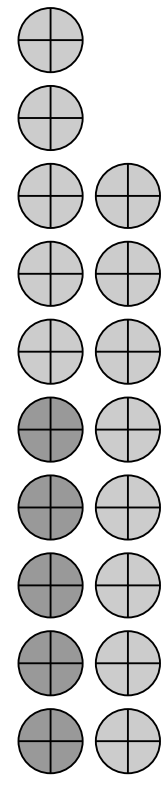

Month 1

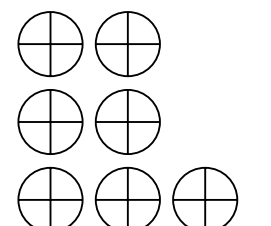
$\oplus \oplus \oplus$ $\oplus \oplus \oplus$ $\oplus \oplus \oplus$ $\oplus \oplus \oplus$ $\ominus \oplus \oplus$ $\ominus \oplus \oplus$ $\oplus \oplus \oplus$

Month 2
Figure 2 Graphical representation of enhancing lesions at baseline, month 1, and month 2. Enhancing lesions present at month 0 are shown in dark grey, new enhancing lesions appearing at month 1 and 2 are shown in light grey and white, respectively.

\section{DISCUSSION}

In this study we aimed to address four questions relating to inflammation in early PPMS. The first and most fundamental question is whether there is an early enhancing phase in PPMS, as there is in RRMS. This study produced no evidence of enhancement as a universal feature in early PPMS. Even when triple dose gadolinium and serial examination was used inflammation was still absent in the majority of cases. However, $42 \%$ of patients had some enhancement, and while this was much less than the $75 \%$ who enhanced in a clinically early RRMS cohort examined under identical circumstances, ${ }^{21}$ it is more than is seen in established PPMS. For example, using entry data from a recent clinical trial in PPMS (where the median time since first symptoms was 10.9 years) $14.1 \%$ of patients had enhancing lesions. ${ }^{7}$ However, a complicating factor is that this clinical trial, together with the majority of studies of enhancement in PPMS, used single rather than triple dose Gd-DTPA. It is still not clear whether the use of triple dose Gd-DTPA leads to detection of a larger number of enhancing lesions in PPMS, as the two small studies that have examined this question drew different conclusions. ${ }^{9}{ }^{10}$

In the spinal cord, little enhancement is seen $(6 \%$ of patients, compared with $33 \%$ of patients in a clinically early RRMS cohort). ${ }^{21}$ Therefore, although a cord presentation is common in PPMS, it does not appear to be associated with inflammation, even though the assessment was made some years after the initial presentation.

The cerebral inflammation seen in early PPMS does not seem to have any distinguishing features. Enhancing lesion number, volume, location, and duration are all similar to those reported in RRMS and SPMS. ${ }^{22}{ }^{23}$ The only previous comparable study in PPMS, using triple dose gadolinium, involved 10 patients with a mean disease duration of 6.5 years and showed very similar results. ${ }^{9}$ The evolution of enhancing lesions into T2 areas of hyperintensity or T1 areas of hypointensity is also similar to that seen in other clinical subgroups. $^{24}$ This suggests that the underlying disease mechanism, at least with respect to focal blood-brain barrier breakdown, may be common to all types of multiple sclerosis.

One of the striking findings in this study was the relative consistency of enhancement among patients. Cord enhancement was not seen in the absence of enhancing lesions in the brain. In addition, in the subset of 13 patients who enhanced at month 0 , eight had further enhancement over the next two months $(69 \%)$, which in the majority was the result of new enhancing lesions.. Finally, all 12 patients who enhanced at six months also had baseline enhancement. This consistency of inflammation among a subset of patients may have implications for treatment, which will be discussed below. 
Patients with enhancing and non-enhancing scans did not differ in presenting history but did on other clinical and MRI measures. Patients with enhancing lesions had greater disability, higher lesion loads, and evidence of greater brain atrophy. Future follow up of this cohort will determine whether enhancement is truly an adverse feature in PPMS.

The presence or absence of enhancement may also reflect important differences in immunopathogenesis within PPMS. In our study all patients had oligoclonal bands in their cerebrospinal fluid, but in a recent clinical trial $21 \%$ of patients did not have oligoclonal bands at entry. In this trial there were strong trends to increased enhancement in patients with oligoclonal bands and an association with higher lesion loads $\left(p=0.1, p<0.001\right.$, respectively). ${ }^{7}$ The presence of oligoclonal bands in all patients in the present study may therefore have increased the likelihood of finding enhancement.

Finally, do the clinical and MRI findings of this study provide any insights into the pathological role of inflammation in PPMS? Inflammation, where present, seems to be associated with adverse features, though it does not necessarily follow that this relation is causal. To date, inflammation suppressing treatments such as interferon beta and glatiramer acetate appear to have little impact on disease progression when used in patients with PPMS. Might a greater clinical effect be seen in patients with evidence of inflammation? This question may be answered by the recently terminated large international trial of glatiramer acetate in PPMS, where single dose gadolinium was given to all subjects. ${ }^{7}$ Equally, the question remains as to the nature of the non-inflammatory mechanism of disability that is operating in the majority of patients who do not show enhancement.

At this point it is relevant to consider a potential limitation of this study-namely, that although patients may be in the early clinical stage of their condition, they may not necessarily be in the early stages of the disease process. The considerable degree of MRI abnormality seen on some of the initial scans, even when the clinical history was short, suggests that this may be the case. This point has been well illustrated in a recent case report of a patient with PPMS who had abnormalities on brain MRI 10 years before the onset of clinical symptoms. ${ }^{25}$ Thus the presence of an inflammatory process in a preclinical phase (which persists into the clinical phase in a proportion of patients) cannot be excluded.

Ongoing follow up of this cohort will provide further data which will help determine whether inflammation is a transient phase in early PPMS (that is, whether cessation of enhancement will be seen in some patients over time as they become clinically established). We will also explore the pathological associations and consequences of enhancement in greater detail using a wider range of newer and pathologically more specific MRI techniques.

\section{ACKNOWLEDGEMENTS}

We would like to acknowledge the support of The Wellcome Trust (GTI), the Spanish Ministry of Health (JSG), and the Multiple Sclerosis Society of Great Britain and Northern Ireland.

\section{Authors' affiliations \\ G T Ingle, J Sastre-Garriga, D H Miller, A J Thompson, Institute of Neurology, University College London, Queen Square, London, UK}

Competing interests: none declared

\section{REFERENCES}

1 Thompson AJ, Polman $\mathrm{CH}$, Miller DH, et al. Primary progressive multiple sclerosis. Brain 1997;120:1085-96.

2 Bashir K, Whitaker JN. Clinical and laboratory features of primary progressive and secondary progressive MS. Neurology 1999;53:765-71.

3 Grossman RI, Gonzalez-Scarano F, Atlas SW, et al. Multiple sclerosis: gadolinium enhancement in MR imaging. Radiology 1986;161:721-5.

4 Katz D, Taubenberger JK, Cannella B, et al. Correlation between magnetic resonance imaging findings and lesion development in chronic, active multiple sclerosis. Ann Neurol 1993;34:661-9.

5 Bruck W, Bitsch A, Kolenda $\mathrm{H}$, et al. Inflammatory central nervous system demyelination: correlation of magnetic resonance imaging findings with lesion pathology. Ann Neurol 1997;42:783-93.

6 Thompson AJ, Kermode AG, Wicks D, et al. Major differences in the dynamics of primary and secondary progressive multiple sclerosis. Ann Neurol 1991;29:53-62.

7 Wolinsky JS. The diagnosis of primary progressive multiple sclerosis. J Neurol Sci 2003;206: 145-52.

8 Kidd D, Thorpe JW, Kendall BE, et al. MRI dynamics of brain and spinal cord in progressive multiple sclerosis. J Neurol Neurosurg Psychiatry 1996;60:15-19.

9 Filippi M, Campi A, Martinelli V, et al. Comparison of triple dose versus standard dose gadolinium-DTPA for detection of MRI enhancing lesions in patients with primary progressive multiple sclerosis. I Neurol Neurosurg Psychiatry 1995;59:540-4.

10 Silver NC, Good CD, Barker GJ, et al. Sensitivity of contrast enhanced MRI in multiple sclerosis. Effects of gadolinium dose, magnetization transfer contrast and delayed imaging. Brain 1997;120:1149-61.

11 Miller DH, Rudge P, Johnson G, et al. Serial gadolinium enhanced magnetic resonance imaging in multiple sclerosis. Brain 1988;111:927-39.

12 Revesz T, Kidd D, Thompson AJ, et al. A comparison of the pathology of primary and secondary progressive multiple sclerosis. Brain 1994; 117:759-65.

13 Leary SM, Miller DH, Stevenson VL, et al. Interferon beta-la in primary progressive MS: an exploratory, randomized, controlled trial. Neurology 2003;60:44-51.

14 Wolinsky JS, Narayana PA, He R. Overview of treatment trials: early baseline clinical MRI data of the PROMISe trial. In: Filippi M, Comi G, eds. Primary progressive multiple sclerosis. Milan: Springer-Verlag Italia, 2002:47-61

15 Rudick RA. Disease-modifying drugs for relapsing-remitting multiple sclerosis and future directions for multiple sclerosis therapeutics. Arch Neurol 1999:56:1079-84.

16 Thompson AJ, Montalban X, Barkhof F, et al. Diagnostic criteria for primary progressive multiple sclerosis: a position paper. Ann Neurol 2000;47:831-5.

17 Cutter GR, Baier ML, Rudick RA, et al. Development of a multiple sclerosis functional composite as a clinical trial outcome measure. Brain 1999; 122:871-82.

18 Losseff NA, Wang L, Lai HM, et al. Progressive cerebral atrophy in multiple sclerosis. A serial MRI study. Brain 1996;119:2009-19.

19 Losseff NA, Webb SL, O'Riordan Jl, et al. Spinal cord atrophy and disability in multiple sclerosis. A new reproducible and sensitive MRI method with potential to monitor disease progression. Brain 1996;119:701-8.

20 Brex PA, Jenkins R, Fox NC, et al. Detection of ventricular enlargement in patients at the earliest clinical stage of MS. Neurology 2000;54:1689-91.

21 Davies GR, Chard DT, Griffin CMB, et al. Triple dose gadolinium enhanced MRI of the brain and spinal cord in early relapsing and remitting multiple sclerosis is predictive of future relapses. I Neurol Neurosurg Psychiatry 2002;73:213-36.

22 Filippi M, Rovaris M, Capra R, et al. A multi-centre longitudinal study comparing the sensitivity of monthly MRI after standard and triple dose gadolinium-DTPA for monitoring disease activity in multiple sclerosis. Implications for phase II clinical trials. Brain 1998;121:2011-20.

23 Silver NC, Good CD, Sormani MP, et al. A modified protocol to improve the detection of enhancing brain and spinal cord lesions in multiple sclerosis. J Neurol 2001;248:215-24.

24 van Waesberghe JH, van Walderveen MA, Castelijns JA, et al. Patterns of lesion development in multiple sclerosis: longitudinal observations with T1weighted spin-echo and magnetization transfer MR. Am J Neuroradiol 1998;19:675-83.

25 McDonnell GV, Cabrera-Gomez J, Calne DB, et al. Clinical presentation of primary progressive multiple sclerosis 10 years after the incidental finding of typical magnetic resonance imaging brain lesions: the subclinical stage of primary progressive multiple sclerosis may last 10 years. Mult Scler 2003;9:204-9. 